\title{
Tratamiento de fracturas de falanges y metacarpianos con tornillos endomedulares compresivos
}

\author{
Macarena Layús, Pablo Pasetto \\ Servicio de Mano y Miembro Superior, Sanatorio Británico, Rosario, Santa Fe, Argentina
}

\begin{abstract}
RESUMEN
Objetivos: Evaluar los resultados clínicos y radiográficos de pacientes con fracturas extrarticulares de falanges y metacarpianos de la mano, tratados mediante osteosíntesis mininvasiva con tornillos endomedulares compresivos retrógrados. Materiales y Métodos: Estudio prospectivo que incluyó a pacientes $>18$ años con fracturas cerradas transversales u oblicuas cortas, extrarticulares, de falanges y metacarpianos tratadas entre noviembre de 2016 y junio de 2019. La serie estaba integrada por 47 pacientes, con 76 fracturas ( 28 de metacarpianos, 27 de falange proximal y 21 de falange media). Se evaluaron los resultados clínicos y funcionales con goniometría, registrando el rango de movilidad activa total. La evaluación radiográfica se realizó sobre la base de los criterios establecidos por Pun y cols. Todos los pacientes completaron el cuestionario QuickDASH en el examen final. Resultados: El tiempo promedio de seguimiento fue de 24.5 meses. El rango de movilidad activa total promedio fue de $238^{\circ}$ para todas las fracturas, $252^{\circ}$ para las de metacarpianos y $230^{\circ}$ para las de falanges. Se observó la consolidación radiográfica de todas las fracturas. El tiempo promedio de retorno a la actividad habitual fue de 79 días. El resultado final del cuestionario QuickDASH fue 4,5. No hubo complicaciones ni cirugías secundarias. Conclusiones: La osteosíntesis mininvasiva con tornillos endomedulares compresivos retrógrados resultó una alternativa eficaz para el tratamiento de fracturas extrarticulares de falanges y metacarpianos.
\end{abstract}

Palabras clave: Fractura; metacarpianos; falanges; tornillos endomedulares compresivos.

Nivel de Evidencia: II

\section{Treatment of phalangeal and metacarpal fractures with intramedullary compression screws}

\section{ABSTRACT}

Objective: To study the clinical and radiological outcomes of patients with extra-articular phalangeal and metacarpal fractures who were treated with minimally invasive internal fixation using retrograde intramedullary compression screws. Materials and Methods: Prospective study in patients over 18 years of age treated for simple, transverse or short oblique, extra-articular phalangeal and metacarpal fractures between November 2016 and June 2019. The series included 47 patients and 76 fractures: 28 metacarpal bones (MC), 27 proximal phalanges (PP), 21 middle phalanges (MP). Clinical and functional outcomes were assessed with goniometry, documenting the total active range of motion (TAM). Radiological outcomes were assessed using the criteria proposed by Pun et al. All patients completed the Quick DASH questionnaire at last follow-up. Results: The average follow-up period was 24.5 months. All study fractures average TAM was $238^{\circ}$, metacarpal TAM was $252^{\circ}$, and phalangeal TAM was $230^{\circ}$. Radiograph evaluation showed fracture consolidation in all cases. Average time taken to return to normal daily activities was 79 days. Average final Quick DASH score was 4.5. There were no complications nor secondary surgeries. Conclusions: Minimally invasive internal fixation with retrograde intramedullary compression screw proved to be a highly effective option in the treatment of extra-articular phalangeal and metacarpal fractures.

Key words: Fracture; metacarpal; phalanx; screws; intramedullary; compression.

Level of Evidence: II

Recibido el 30-7-2020. Aceptado luego de la evaluación el 2-8-2020 • Dra. MACARENA LAYÚS • layusmac@ gmail.com (ID https://orcid.org/0000-0003-3852-5155 Cómo citar este artículo: Layús M, Pasetto P. Tratamiento de fracturas de falanges y metacarpianos con tornillos endomedulares compresivos. Rev Asoc Argent Ortop Traumatol 2020;85(Supl.):S2-S11-. https://doi.org/10.15417/issn.1852-7434.2020.85.4S.1172 


\section{INTRODUCCIÓN}

Las fracturas de metacarpianos y falanges en las manos son frecuentes y la complejidad de su tratamiento varía. La finalidad del tratamiento, incruento o cruento, es lograr una óptima recuperación funcional de la mano, con alineación aceptable de las fracturas, consolidación ósea estable y movilidad sin restricciones. ${ }^{1}$ Se han descrito múltiples procedimientos con este fin. Las fracturas estables pueden tratarse satisfactoriamente con férulas y movilización temprana controlada. La elección del tipo de tratamiento para las fracturas inestables depende, en gran medida, del tipo y la localización del trazo de fractura. Los tratamientos más frecuentes para las fracturas extrarticulares son: osteosíntesis percutánea con clavijas de Kirschner y fijación con placas y tornillos. ${ }^{2}$ En la actualidad, no hay consenso sobre el mejor método terapéutico.

La movilización temprana tiene especial importancia, ya que la recuperación de los tejidos blandos puede ser más problemática que la consolidación ósea. ${ }^{3}$

En los últimos años, se ha descrito el uso de tornillos endomedulares compresivos retrógrados (TECR) por técnica mininvasiva, que representa una opción válida y permite una movilización temprana. ${ }^{2}$ Se ha evaluado el compromiso de la superficie articular en el sitio de ingreso del tornillo mediante estudios cadavéricos y análisis de tomografías computarizadas 3D que avalan su uso., ${ }^{4,5}$

El objetivo de este estudio es comunicar los resultados clínicos y radiográficos de pacientes con fracturas extrarticulares de falanges y metacarpianos de la mano, tratados mediante osteosíntesis mininvasiva con TECR.

\section{MATERIALES Y MÉTODOS}

Se llevó a cabo un estudio prospectivo no aleatorizado entre noviembre de 2016 y junio de 2019. Se obtuvo el consentimiento informado.

Los criterios de inclusión fueron: 1) pacientes $>18$ años, 2) fracturas cerradas de metacarpianos, falange proximal y falange media transversales u oblicuas cortas, 3) sin compromiso articular, 4) seguimiento mínimo de 12 meses.

Los criterios de exclusión fueron: 1) fracturas oblicuas largas, espiroideas o articulares, 2) fracturas previas en la misma mano y 3 ) lesiones asociadas (tendinosas, nerviosas, vasculares, de partes blandas).

La serie estaba integrada por 47 pacientes ( 45 hombres y 2 mujeres), con 76 fracturas ( 28 de metacarpianos, 27 de falange proximal y 21 de falange media). Cuarenta y cinco eran transversales; 16 , oblicuas cortas; 15 presentaron un tercer fragmento (Figura 1, Tabla 1).

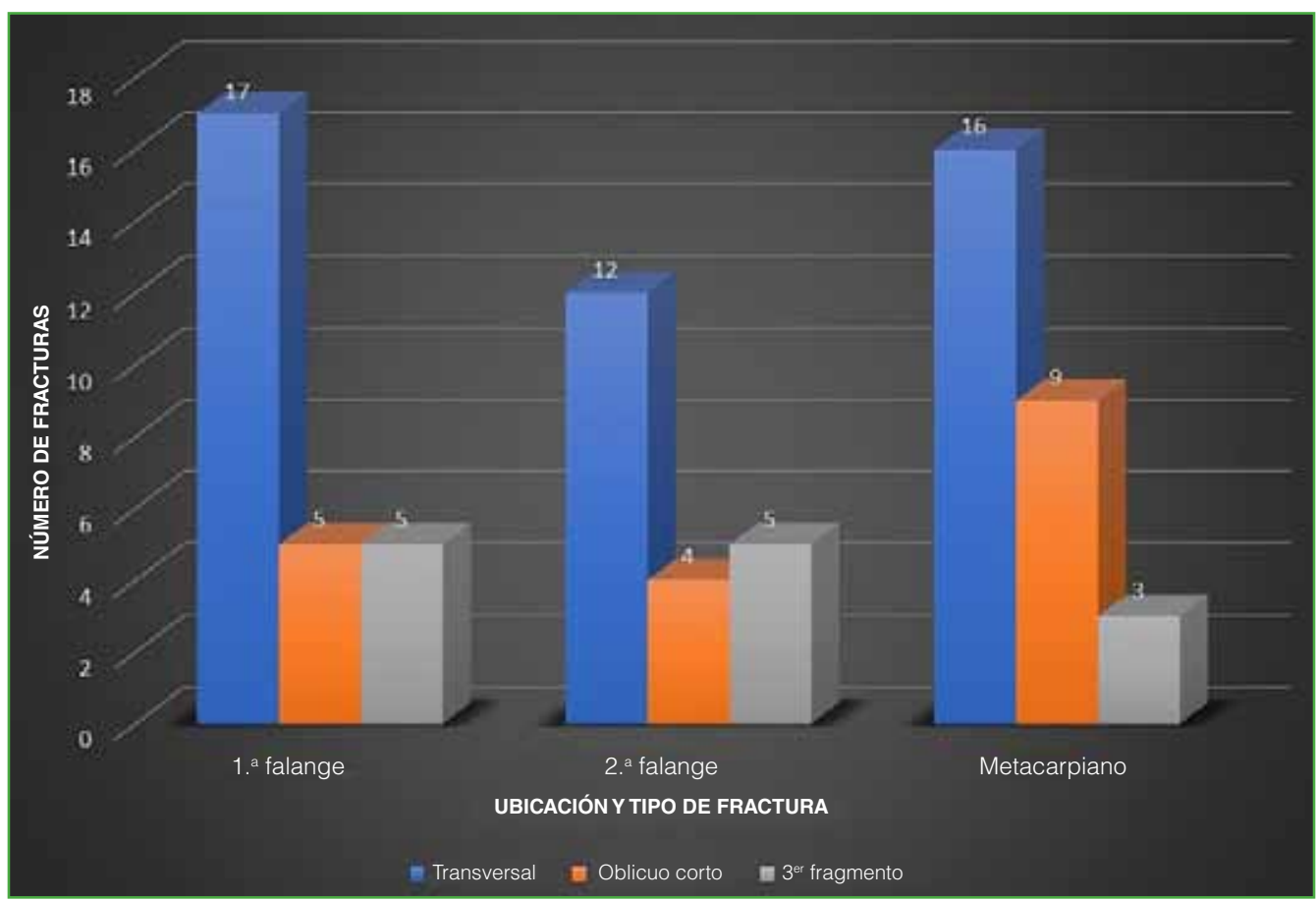

Figura 1. Distribución según la ubicación y el tipo de fracturas de metacarpianos y falanges en los pacientes de esta serie. 
Tabla 1. Serie de pacientes operados con tornillos endomedulares compresivos

\begin{tabular}{|c|c|c|c|c|}
\hline Pacientes & Edad (años) & Cantidad de fracturas & Ubicación & Tornillo (mm) \\
\hline 1 & 38 & 1 & F2 D3 & 2,3 \\
\hline 2 & 40 & 2 & F2 D3-4 & 2,3 \\
\hline 3 & 20 & 4 & F1 D1-2-3, F2 D3 & 2,0 \\
\hline 4 & 30 & 2 & F1 D2-3 & 2,0 \\
\hline 5 & 21 & 2 & MTC 3-4 & 3,0 \\
\hline 6 & 39 & 1 & MTC 5 & 3,0 \\
\hline 7 & 49 & 1 & MTC 5 & $3,0 \mathrm{RC}$ \\
\hline 8 & 20 & 1 & MTC 5 & $3,0 \mathrm{RC}$ \\
\hline 9 & 31 & 1 & MTC 5 & 3,2 \\
\hline 10 & 29 & 1 & MTC 5 & 3,0 \\
\hline 11 & 24 & 1 & F1 D2 & 2,0 \\
\hline 12 & 31 & 2 & MTC 4-5 & 2,7 \\
\hline 13 & 28 & 4 & F1 D1-2, F2 D2-3 & 2,3 \\
\hline 14 & 24 & 1 & F1 D1 & 2,3 \\
\hline 15 & 18 & 1 & MTC 5 & 3,0 \\
\hline 16 & 32 & 1 & MTC 5 & $3,0 \mathrm{RC}$ \\
\hline 17 & 34 & 2 & F2 D2-3 & 2,0 \\
\hline 18 & 35 & 3 & MTC 3-4-5 & 3,0 \\
\hline 19 & 53 & 1 & MTC 5 & 3,0 \\
\hline 20 & 32 & 3 & F1 D3-4-5 & 2,0 \\
\hline 21 & 22 & 2 & F2 D3-4 & 2,0 \\
\hline 22 & 64 & 1 & F1 D5 & 2,0 \\
\hline 23 & 34 & 1 & F2 D3 & 2,0 \\
\hline 24 & 26 & 2 & F2 D2-3 & 2,0 \\
\hline 25 & 25 & 3 & F1 D1, F2 D2-3 & 2,4 \\
\hline 26 & 34 & 1 & MTC 5 & 3,0 \\
\hline 27 & 31 & 3 & F1 D1, F2 D2-3 & 2,4 \\
\hline 28 & 32 & 2 & F1 D2-3 & 2,4 \\
\hline 29 & 31 & 1 & MTC 5 & 3 \\
\hline 30 & 34 & 1 & F1 D1 & 2,4 \\
\hline 31 & 24 & 1 & F2 D2 & 2 \\
\hline 32 & 48 & 1 & MTC 5 & 3 \\
\hline 33 & 31 & 3 & MTC 2, F1 D3-D4 & 3 \\
\hline 34 & 38 & 2 & MTC 4-5 & 3 \\
\hline 35 & 21 & 1 & MTC 2 & 3 \\
\hline 36 & 24 & 1 & MTC 5 & 3 \\
\hline 37 & 20 & 1 & MTC 4 & 3 \\
\hline 38 & 29 & 2 & MTC 4-5 & 3 \\
\hline 39 & 26 & 3 & F1 D2-3-4 & 2,4 \\
\hline 40 & 22 & 2 & F2 D3-4 & 2 \\
\hline 41 & 23 & 1 & F1 D1 & 2,4 \\
\hline 42 & 21 & 1 & F2 D3 & 2 \\
\hline 43 & 51 & 1 & F1 D5 & 2,4 \\
\hline 44 & 25 & 1 & MTC 5 & 3 \\
\hline 45 & 25 & 1 & F1 D5 & 2,4 \\
\hline 46 & 30 & 1 & MTC 5 & 3 \\
\hline 47 & 34 & 1 & F1 D5 & 2,4 \\
\hline
\end{tabular}

$\mathrm{D}=$ dedo, $\mathrm{MTC}=$ metacarpiano, $\mathrm{F} 1=$ falange proximal, $\mathrm{F} 2=$ falange media, $\mathrm{F} 3=$ falange distal, $\mathrm{RC}=$ rosca completa 
Veintidós pacientes tenían múltiples fracturas en la misma mano. El promedio de edad era de 31 años (rango 18-64). En la mayoría (33 pacientes), el trauma se relacionó con la actividad laboral. La intervención quirúrgica se practicó, en promedio, a los 6.7 días del trauma (rango 3-20). Solo dos especialistas estuvieron a cargo de los procedimientos. Todos los pacientes utilizaron una férula de Zimmer o de yeso posoperatoria hasta el retiro de los puntos de sutura (10-12 días) y comenzaron la movilidad temprana con un terapista ocupacional entre los 5 y 7 días posteriores a la cirugía. Se registró el tiempo de retorno a la actividad habitual (laboral/deportiva). Se establecieron protocolos de evaluación objetiva mediante goniometría y radiografías. Se evaluaron los resultados clínicos y funcionales con goniometría, registrando el rango de movilidad activa total definido como la suma de la flexión activa de las articulaciones metacarpofalángica, interfalángica proximal y distal, menos el déficit de extensión de dichas articulaciones. ${ }^{6}$ Los resultados se consideraron excelentes si el valor era $>150^{\circ}$, buenos de $125^{\circ}$ a $149^{\circ}$, regulares de $90^{\circ}$ a $124^{\circ}$ y malos $<90^{\circ}$.

Se evaluaron las radiografías de frente y de perfil tomadas antes de la cirugía, en el posoperatorio inmediato y al final del seguimiento. Se consideró que un resultado era satisfactorio si cumplía con los criterios establecidos por Pun y cols. ${ }^{7}$ En las fracturas de falange, se consideró tolerable un ángulo $<10^{\circ}$ en los planos coronal y sagital, sin rotación y, en las fracturas de metacarpianos, un acortamiento $<6 \mathrm{~mm}$ sin rotación ni angulación de la cabeza. Se utilizó el cuestionario QuickDASH para la evaluación subjetiva al final del seguimiento. ${ }^{8}$

\section{Técnica quirúrgica}

Se coloca al paciente en decúbito dorsal, con el miembro superior en la mesa quirúrgica. Se administra anestesia plexual o asociación de neuroleptoanestesia y anestesia local troncular. Se efectúa una reducción manual controlada bajo radioscopia. Para las fracturas de metacarpianos, se realiza una flexión máxima de la falange proximal, exponiendo la cabeza del metacarpiano. Se hace una incisión transversal de $1 \mathrm{~cm}$, en dicha zona, con una pequeña apertura longitudinal del tendón extensor en la zona central. Se coloca, bajo guía radioscópica, una clavija de Kirschner de 1,0 mm, retrógrada siguiendo el canal endomedular; se continúa con el fresado y la colocación del tornillo de doble rosca canulado, autocompresivo de $3,0 \mathrm{~mm}$, según la técnica habitual, asegurando que la cabeza del tornillo quede completamente por debajo de la línea condral (Figura 2). La longitud máxima disponible de estos tornillos es $40 \mathrm{~mm}$.

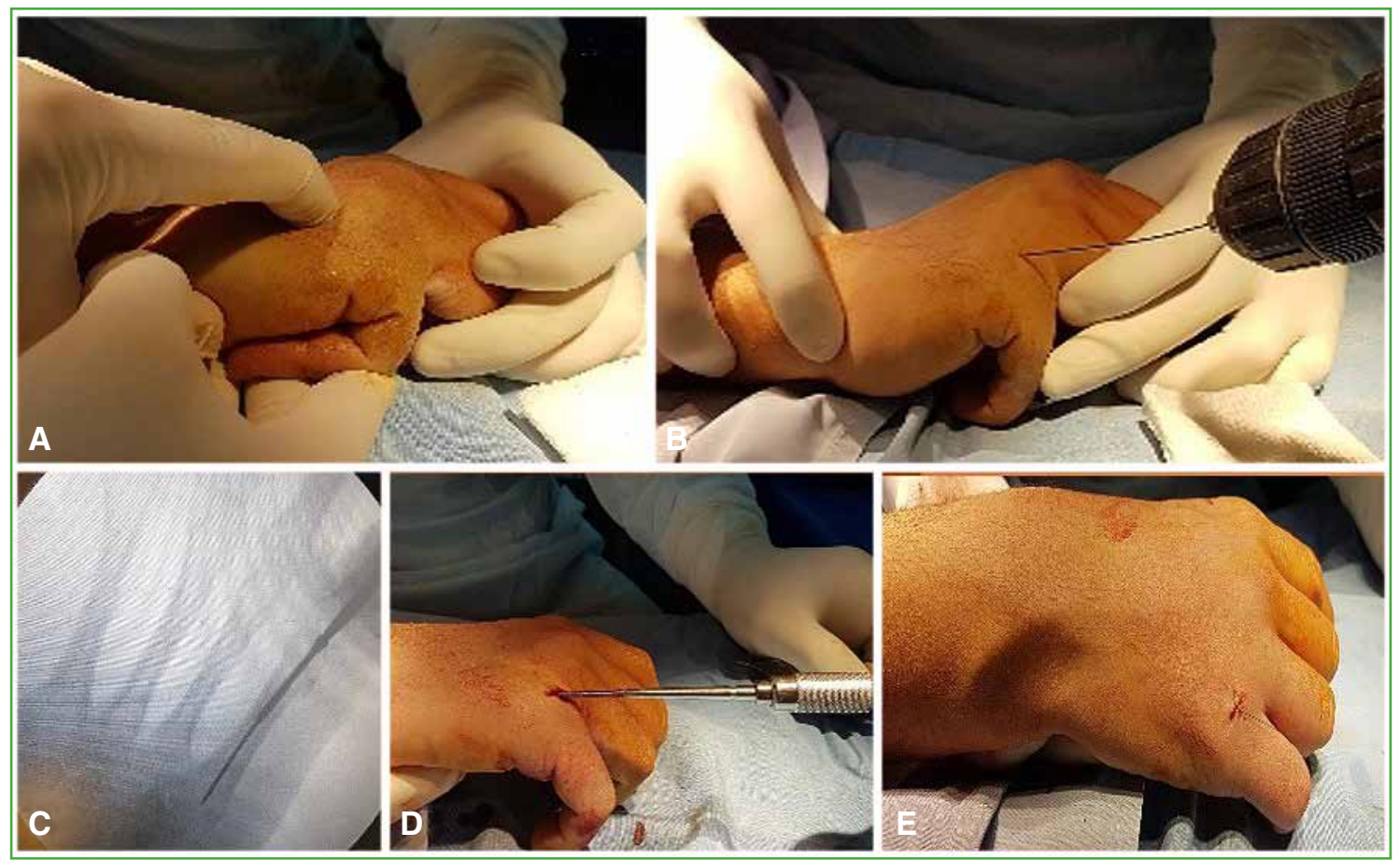

Figura 2. Técnica mininvasiva con tornillo endomedular compresivo retrógrado para fractura de metacarpianos. A. Maniobra de reducción cerrada. B. Colocación de clavija endomedular retrógrada de $1 \mathrm{~mm}$ y fresado únicamente del sitio de entrada en la cabeza del metacarpiano, a través de un abordaje transversal y una pequeña sección longitudinal del tendón extensor. C y D. Inserción del tornillo canulado compresivo. E. Cierre de la herida. 
El procedimiento para fracturas de falange proximal es similar. Se realiza una flexión máxima de la articulación interfalángica proximal y un abordaje transversal de $1 \mathrm{~cm}$ con una pequeña apertura del tendón extensor, exponiendo la cabeza de la falange proximal. Se utilizan tornillos de 2,2 $\mathrm{mm}$. La longitud máxima disponible de estos tornillos es $34 \mathrm{~mm}$.

En los casos de fractura de falange media, el abordaje se realiza sobre la articulación interfalángica distal, con flexión máxima de dicha articulación para exponer la cabeza de la falange media (Figura 3). Se utilizan también tornillos de $2,2 \mathrm{~mm}$.

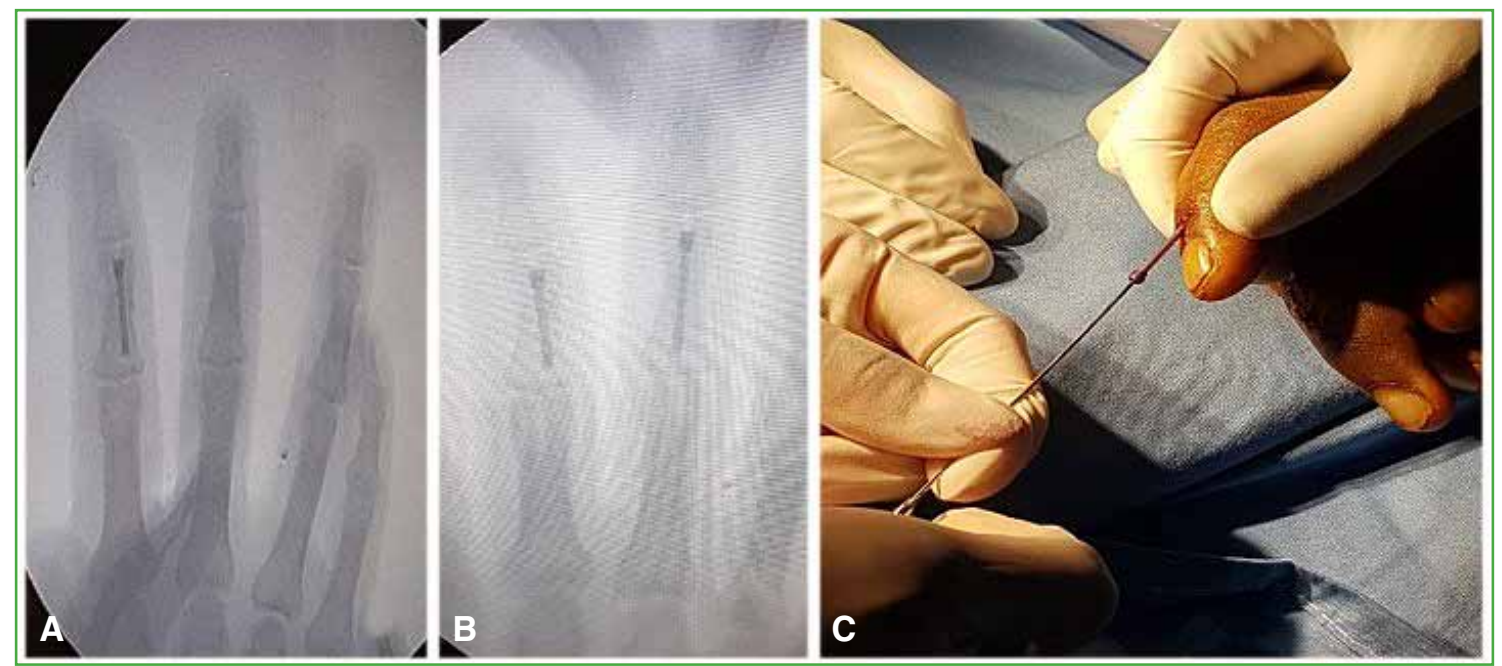

Figura 3. Técnica mininvasiva para fractura de falange media. A y B. Imagen radioscópica de tornillos en la falange media. C. Colocación de tornillos canulados retrógrados.

Durante la colocación del tornillo compresivo, es importante controlar la rotación mediante una compresión lateral y la ausencia de solapamientos digitales al simular el cierre de puño.

\section{RESULTADOS}

El tiempo promedio de seguimiento fue de 24.5 meses (rango 12-43). La movilidad activa total promedio fue de $238^{\circ}$ (rango 110-280) para todas las fracturas, $253^{\circ}$ (rango 205-280) para fracturas de metacarpianos y $229^{\circ}$ (rango 110-280) para las de falanges. El tiempo promedio de retorno a la actividad habitual fue de 79 días (rango 21-120). En la evaluación final, todas las fracturas tenían una evolución radiográfica satisfactoria. El resultado final del cuestionario QuickDASH fue 4,5 (rango 0-5). No se registraron complicaciones. Ningún paciente requirió una cirugía secundaria (Tablas 2 y 3). A modo de ejemplo, se presentan casos completos resueltos de fracturas de falanges (Figura 4) y de metacarpianos (Figura 5).

\section{DISCUSIÓN}

Hebert y Fisher popularizaron el uso de tornillos canulados compresivos sin cabeza, principalmente para la estabilización de fracturas de escafoides. ${ }^{9}$

En los últimos años, diversas publicaciones han comunicado su uso para tratar fracturas extrarticulares de falanges y metacarpianos..$^{2,10,11}$

Del Piñal y cols. describieron el empleo de dichos tornillos para la osteosíntesis de fracturas extrarticulares de falanges y metacarpianos con técnica mininvasiva retrógrada, y destacan como principal ventaja de esta técnica su capacidad para lograr una fijación estable que permite la movilización temprana y evita la rigidez articular metacarpofalángica e interfalángica que, por lo general, se produce con otro tipo de tratamientos. ${ }^{2}$ 
Tabla 2. Resultados de la serie

\begin{tabular}{|c|c|c|c|}
\hline Paciente & Retorno a la actividad (días) & Cuestionario QuickDASH & Seguimiento (meses) \\
\hline 1 & 92 & 5 & 43 \\
\hline 2 & 95 & 4 & 41 \\
\hline 3 & 115 & 5 & 33 \\
\hline 4 & 90 & 4 & 33 \\
\hline 5 & 95 & 5 & 33 \\
\hline 6 & 86 & 5 & 32 \\
\hline 7 & 21 & 0 & 32 \\
\hline 8 & 35 & 5 & 30 \\
\hline 9 & 90 & 4 & 30 \\
\hline 10 & 95 & 5 & 30 \\
\hline 11 & 85 & 4 & 29 \\
\hline 12 & 60 & 5 & 29 \\
\hline 13 & 94 & 4 & 28 \\
\hline 14 & 67 & 5 & 28 \\
\hline 15 & 55 & 4 & 27 \\
\hline 16 & 68 & 5 & 27 \\
\hline 17 & 95 & 5 & 27 \\
\hline 18 & 70 & 5 & 26 \\
\hline 19 & 67 & 5 & 26 \\
\hline 20 & 95 & 4 & 26 \\
\hline 21 & 95 & 5 & 26 \\
\hline 22 & 90 & 5 & 26 \\
\hline 23 & 55 & 5 & 25 \\
\hline 24 & 105 & 4 & 25 \\
\hline 25 & 85 & 5 & 25 \\
\hline 26 & 55 & 4 & 25 \\
\hline 27 & 95 & 5 & 25 \\
\hline 28 & 90 & 5 & 25 \\
\hline 29 & 67 & 4 & 23 \\
\hline 30 & 70 & 5 & 22 \\
\hline 31 & 77 & 4 & 21 \\
\hline 32 & 65 & 4 & 20 \\
\hline 33 & 90 & 5 & 18 \\
\hline 34 & 72 & 5 & 18 \\
\hline 35 & 69 & 5 & 16 \\
\hline 36 & 65 & 5 & 16 \\
\hline 37 & 70 & 5 & 15 \\
\hline 38 & 75 & 4 & 15 \\
\hline 39 & 98 & 4 & 15 \\
\hline 40 & 78 & 5 & 14 \\
\hline 41 & 90 & 5 & 14 \\
\hline 42 & 80 & 5 & 14 \\
\hline 43 & 75 & 5 & 13 \\
\hline 44 & 68 & 5 & 13 \\
\hline 45 & 75 & 4 & 13 \\
\hline 46 & 70 & 5 & 12 \\
\hline 47 & 82 & 4 & 12 \\
\hline
\end{tabular}


Tabla 3. Resultados del rango de movilidad activa total

\begin{tabular}{|l|c|c|}
\hline Ubicación & Rango de movilidad activa total (promedio) & Rango \\
\hline Total de fracturas & $238^{\circ}$ & $110-280^{\circ}$ \\
\hline Metacarpianos & $253^{\circ}$ & $205-280^{\circ}$ \\
\hline Falanges & $229^{\circ}$ & $110-280^{\circ}$ \\
\hline
\end{tabular}
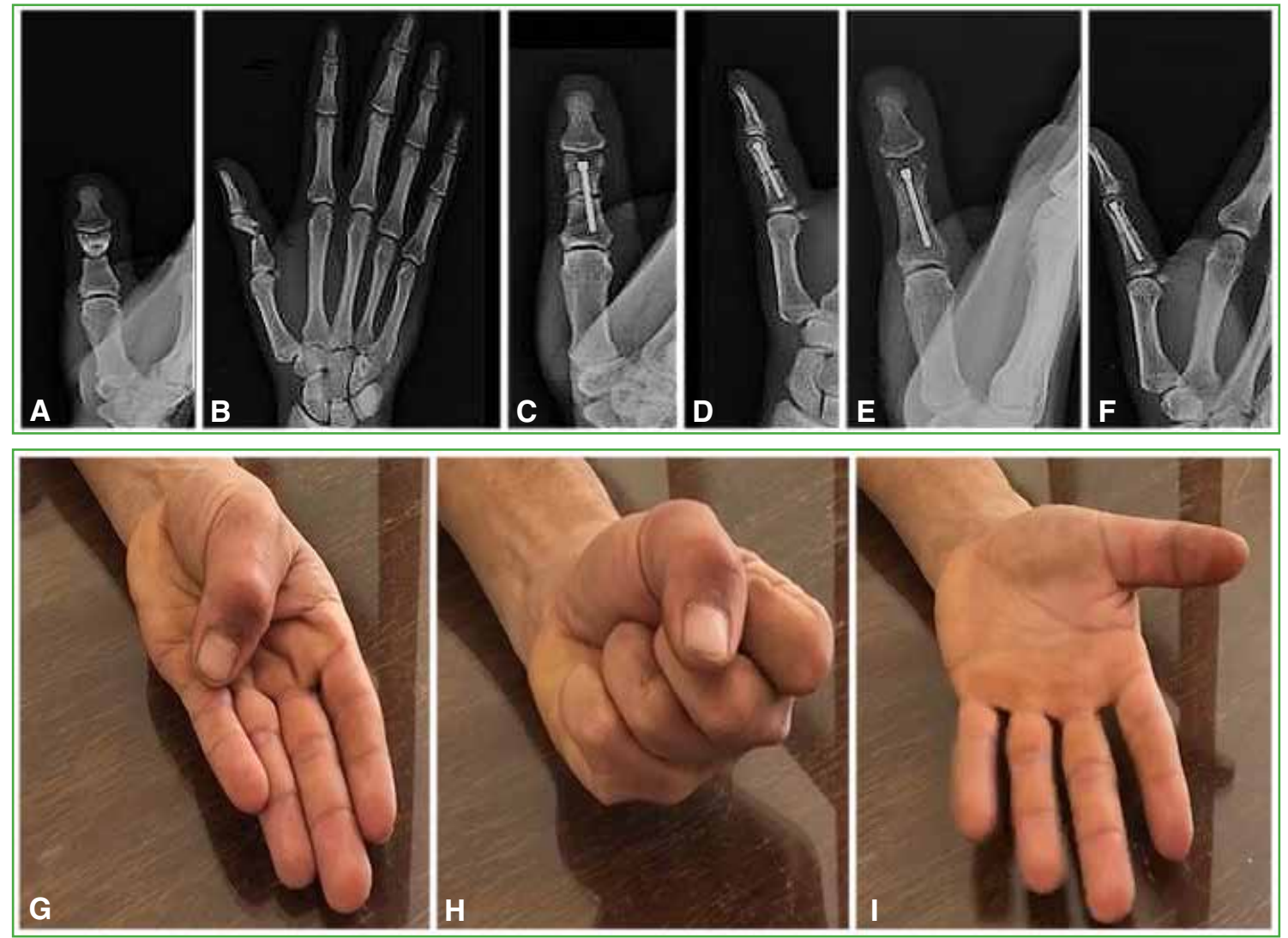

Figura 4. Paciente con fractura de falange proximal de pulgar. A y B. Radiografías de perfil y de frente preoperatorias. C y D. Par radiográfico al mes de la cirugía. E y F. Control a los tres meses de la cirugía. G-I. Rango de movilidad a los tres meses de la operación.

La osteosíntesis con placas y tornillos brinda una excelente estabilidad, pero requiere una mayor disección de tejidos blandos, desperiostización que, a menudo, provoca adherencia tendinosa y cicatricial. ${ }^{12,13}$ En una serie de 129 pacientes con 157 fracturas de metacarpianos tratadas mediante reducción abierta y osteosíntesis con placa y tornillos, Fusetti y cols. reportaron complicaciones en más de un tercio de los pacientes, que incluían retraso de la consolidación, adherencias tendinosas y de partes blandas, síndrome de dolor regional complejo e infección profunda. $^{14}$ 

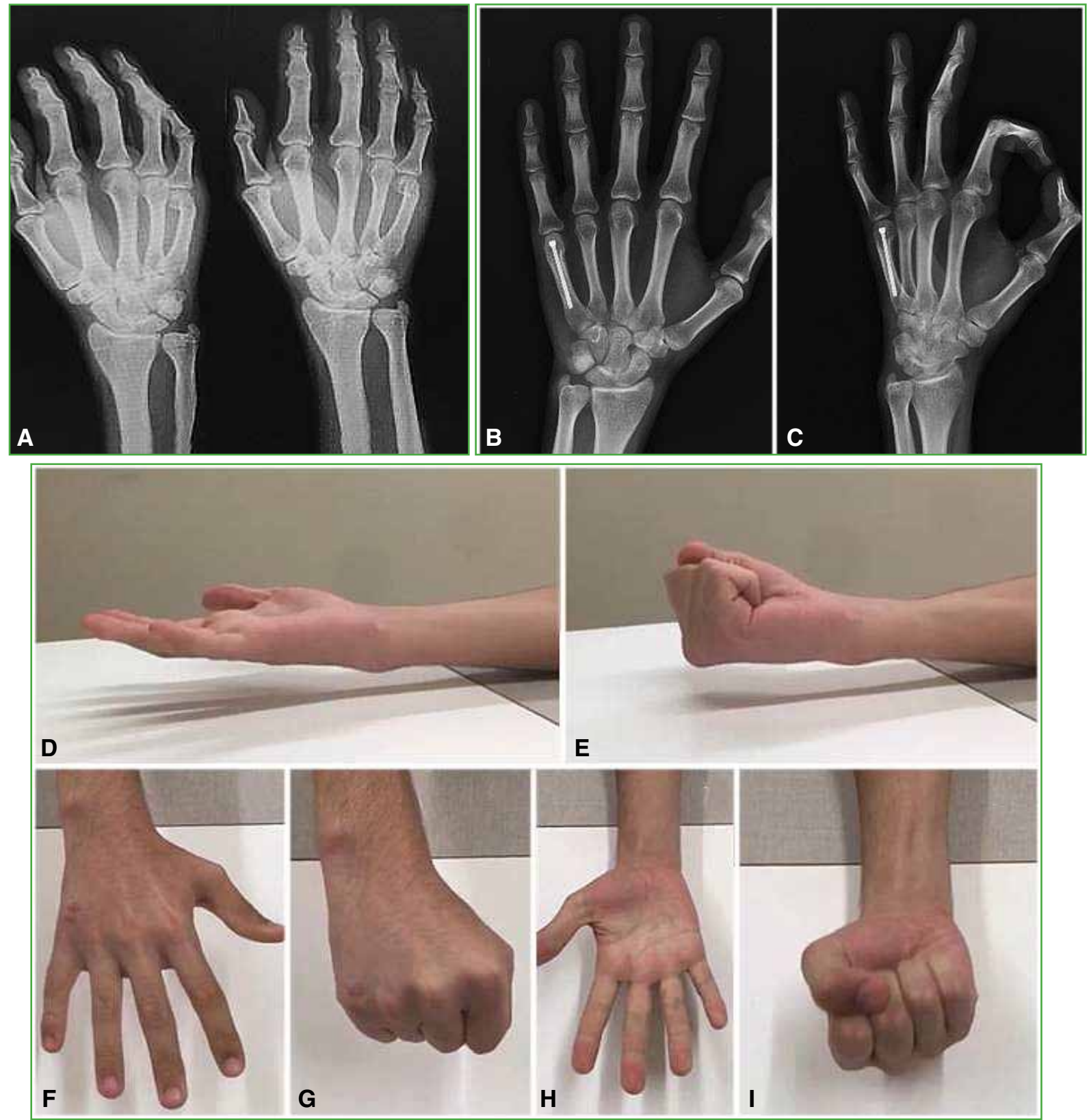

Figura 5. Paciente con fractura del $5^{\text {to }}$ metacarpiano de la mano. A. Radiografías de frente y oblicua preoperatorias. B y C. Par radiográfico a los 45 días de la cirugía. D-I. Resultado funcional a los 60 días de la operación.

La osteosíntesis percutánea con clavijas de Kirschner, si bien evita la exposición del foco fracturario y la disección de partes blandas, requiere de tres a cuatro semanas de inmovilización posoperatoria para reducir, al mínimo, el riesgo de infección superficial o profunda de las clavijas, lo que desencadena su retiro prematuro y procedimientos adicionales. ${ }^{11}$ Hsu y cols. describen, en una serie de 189 pacientes con fracturas de mano y muñeca tratados mediante osteosíntesis percutáneas con clavijas, un 14\% de complicaciones menores (infección superficial, edema, migración de las clavijas) y un 3\% de complicaciones mayores (seudoartrosis, infección profunda). ${ }^{15}$ 
Berg y cols. publicaron un análisis cuantitativo tomográfico computarizado 3D para evaluar el sitio de entrada articular, la superficie articular y el volumen subcondral utilizado en la osteosíntesis de fracturas de cuello de metacarpianos con TECR. Dicho estudio sugiere que la directa visualización del sitio de entrada elimina potencialmente la necesidad de múltiples intentos para conseguir esta premisa, lo cual suele ocurrir durante la osteosíntesis con clavijas percutáneas. Establece que el punto de entrada articular dorsal se encuentra alineado con el canal endomedular y evita el acoplamiento del centro de la base articular a través de la mayor parte del arco del plano sagital. Cumpliendo estos requisitos, la superficie articular de la cabeza del metacarpiano y el volumen subcondral ocupado por el tornillo compresivo son mínimos. ${ }^{5}$

En un estudio cadavérico, Borbas y cols. establecieron que el daño de la superficie articular causado en el punto de entrada del tornillo en las articulaciones metacarpofalángica e interfalángica proximal es menor que el previamente establecido, cuantificado en $8,5 \%$ (tornillos de 3,0 $\mathrm{mm}$ ) y 4,6\% (tornillos de 2,2 $\mathrm{mm}$ ) en promedio, respectivamente. ${ }^{4}$

No existen, hasta el momento, publicaciones que describan el impacto a largo plazo del sitio de entrada del tornillo sobre la superficie articular.

Las fortalezas de nuestro estudio son: el diseño prospectivo, la reproducibilidad de la técnica, el número de pacientes incluidos y la homogeneidad de la enfermedad estudiada. Como debilidades, consideramos la falta de aleatorización de la muestra y el acotado tiempo de seguimiento para evaluar potenciales cambios artrósicos articulares atribuidos al sitio de entrada del tornillo. Una futura revaluación de la misma serie de pacientes con análisis tomográfico de las superficies articulares afectadas aportaría claridad respecto a esta coyuntura.

\section{CONCLUSIONES}

La osteosíntesis mininvasiva con TECR resultó una alternativa eficaz para el tratamiento de fracturas extrarticulares de falanges y metacarpianos.

Conflicto de intereses: Los autores no declaran conflictos de intereses.

\section{BIBLIOGRAFÍA}

1. Kozin SH, Thoder JJ, Lieberman G. Operative treatment of metacarpal and phalangeal shaft fractures. J Am Acad Orthop Surg 2000;8(2):111-21. https://doi.org/10.5435/00124635-200003000-00005

2. Del Piñal F, Moraleda E, Ruas JS, de Piero GH, Cerezal L. Minimally invasive fixation of fractures of the phalanges and metacarpals with intramedullary cannulated headless compression screws. J Hand Surg Am 2015;40:692-700. https://doi.org/10.1016/j.jhsa.2014.11.023

3. Meals C, Meals R. Hand fractures: A review of current treatment strategies. J Hand Surg Am 2013;38(5):1021-31. https://doi.org/10.1016/j.jhsa.2013.02.017

4. Borbas P, Dreu M, Poggetti A, Calcagni M, Giesen T. Treatment of proximal phalangeal fractures with an antegrade intramedullary screw: a cadaver study. J Hand Surg Eur 2016;41(7):683-7. https://doi.org/10.1177/1753193416641319

5. Berg P, Mudgal C, Leibman M, Belsky M, Ruchelsman D. Quantitative 3-dimensional CT analyses of intramedullary headless screw fixation for metacarpal neck fractures. J Hand Surg Am 2013;38(2):322-30. https://doi.org/10.1016/j.jhsa.2012.09.029

6. American Society for Surgery of the Hand. The hand, examination and diagnosis. $3^{\text {rd }}$ ed. New York: Churchill Livingstone; 1990.

7. Pun W, Chow S, So Y, Luk K, Ip F, Chan K, et al. A prospective study on 284 digital fractures of the hand. $J$ Hand Surg Am 1989;14(3):474-81. https://doi.org/10.1016/s0363-5023(89)80006-1 
8. Beaton DE, Wright JG, Katz JN, Upper Extremity Collaborative Group. Development of the QuickDASH: comparison of three item-reduction approaches. J Bone Joint Surg Am 2005;87(5):1038-46. https://doi.org/10.2106/JBJS.D.02060

9. Herbert TJ, Fisher WE. Management of the fractured scaphoid using a new bone screw. J Bone Joint Surg Br 1984;66(1):114-23. PMID: 6693468

10. Boulton CL, Salzler M, Mudgal CS. Intramedullary cannulated headless screw fixation of a comminuted subcapital metacarpal fracture: case report. J Hand Surg Am 2010;35(8):1260-3. https://doi.org/10.1016/j.jhsa.2010.04.032

11. Ruchelsman DE, Puri S, Feinberg-Zadek N, Leibman MI, Belsky MR. Clinical outcomes of limited-open retrograde intramedullary headless screw fixation of metacarpal fractures. J Hand Surg Am 2014;39(12):2390-5. https://doi.org/10.1016/j.jhsa.2014.08.016

12. Ozer K, Gillani S, Williams A, Peterson S, Morgan S. Comparison of intramedullary nailing versus plate-screw fixation of extra-articular metacarpal fractures. J Hand Surg Am 2008;33(10):1724-31. https://doi.org/10.1016/j.jhsa.2008.07.011

13. Ouellette EA, Freeland AE. Use of the minicondylar plate in metacarpal and phalangeal fractures. Clin Orthop Relat Res 1996;327:38-46. https://doi.org/10.1097/00003086-199606000-00007

14. Fusetti C, Meyer H, Borisch N, Stern R, Della Santa D, Papaloïzos M. Complications of plate fixation in metacarpal fractures. J Trauma 2002;52(3):535-9. https://doi.org/10.1097/00005373-200203000-00019

15. Hsu L, Schwartz E, Kalainov D, Chen F, Makowiec R. Complications of K-wire fixation in procedures involving the hand and wrist. J Hand Surg Am 2011;36(4):610-6. https://doi.org/10.1016/j.jhsa.2011.01.023 